\title{
Simulation of droplet dispersion in COVID-19 type pandemics on Fugaku
}

\author{
Rahul Bale* \\ rahul.bale@riken.jp \\ RIKEN Center for Computational \\ Science \\ Kobe, Hyogo, Japan \\ Akiyoshi Iida \\ Toyohashi Institute of Technology \\ Toyohashi, Aichi, Japan
}

\author{
Chung-Gang Li \\ Kobe University \& \\ RIKEN Center for Computational \\ Science \\ Kobe, Hyogo, Japan \\ Ryoichi Kurose \\ Kyoto University \\ Kyoto , Kyoto, Japan
}

\author{
Masashi Yamakawa \\ Kyoto Institute of Technology \\ Kyoto, Kyoto, Japan
}

\author{
Makoto Tsubokura \\ Kobe University \& \\ RIKEN Center for Computational \\ Science \\ Kobe, Hyogo, Japan
}

\begin{abstract}
Transmission of infectious respiratory diseases through airborne dispersion of viruses poses a great risk to public health. In several major diseases, one of the main modes of transmission is through respiratory droplets. Virus laden respiratory droplets and aerosols can be generated during coughing, sneezing and speaking. These droplets and aerosols can remain suspended in air and be transported by airflow posing a risk of infection in individuals who might come in contact with them. With this background, in this work, we present a numerical framework for simulation of dispersion of respiratory sputum droplets using implicit large-eddy simulations. A combination of discrete Lagrangian droplet model and fully compressible Navier-Stokes flow solver is employed in this study. The method is applied to analyze cases such as droplet dispersion during speech and cough under different environmental settings. Furthermore, the performance of the numerical framework is evaluated through strong and weak scaling analysis.
\end{abstract}

\section{CCS CONCEPTS}

- Applied computing $\rightarrow$ Engineering; • Computing methodologies $\rightarrow$ Continuous models.

\section{KEYWORDS}

COVID-19; Respiratory Droplets; Building Cube Method https://doi.org/10.1145/3468267.3470575

\section{INTRODUCTION}

Airborne dispersion of viruses laden sputum droplets can result in airborne disease transmission poses a great risk to public health.

\footnotetext{
${ }^{*}$ Corresponding author.
}

Permission to make digital or hard copies of all or part of this work for personal or classroom use is granted without fee provided that copies are not made or distributed for profit or commercial advantage and that copies bear this notice and the full citation on the first page. Copyrights for components of this work owned by others than the author(s) must be honored. Abstracting with credit is permitted. To copy otherwise, or republish, to post on servers or to redistribute to lists, requires prior specific permission and/or a fee. Request permissions from permissions@acm.org.

PASC '21, July 5-9, 2021, Geneva, Switzerland

(c) 2021 Copyright held by the owner/author(s). Publication rights licensed to ACM ACM ISBN 978-1-4503-8563-3/21/07 . \$ \$15.00

https://doi.org/10.1145/3468267.3470575
While some airborne diseases such as the common cold and seasonal influenza are not very severe, in recent decades several highly infectious and communicable diseases have emerged. Some of these include Severe Acute Respiratory Syndrome (SARS), Middle East Respiratory Syndrome (MERS), H1N1 influenza and the Coronavirus Disease 2019 (COVID-19)[8, 9, 28, 30, 31]. Since its emergence in December 2019, COVID-19 has rapidly transformed from a local epidemic into a global pandemic. It is hypothesized that the primary reason for such a rapid spread of the disease is due to airborne transmission of the Coronavirus through sputum droplets that are ejected during coughing, sneezing and speaking.

In this work, we investigate the dispersion of sputum droplets due to coughing and speaking to better understand the mechanisms of dispersion under different indoor environmental conditions. To that end, we carried out numerical flow simulations in a compressible framework to model the droplet dispersion. While it is possible to adopt an incompressible framework to model droplet dispersion $[17,26]$, in such frameworks the vapour phase of the droplets is not modelled and it's effect ignored. We adopt a fully compressible Navier-Stokes solver wherein the evaporated phase of the droplet and its effect on droplet evaporation is modelled $[3,15]$. A discrete Lagrangian droplet model was used to model droplet transport and evaporation. The droplet model is tied to the flow equations to enable a weak two-way coupling between the droplets and the flow, wherein the droplets are influenced by the flow while the reverse is not allowed, however, the vapour phase of the droplets are influenced by the droplet evaporation. Analysis of the droplet dispersion during cough and speech in a quiescent environment and while speaking in an outdoor environment is carried out in this work.

\section{NUMERICAL FRAMEWORK}

\subsection{Governing Equations}

The numerical formulation employed for modeling droplet dispersion in this work comprises a combination of a Lagrangian and an Eulerian frame of reference. The discretized equations of mass, momentum and energy conservation reside on the Eulerian meshed. The equations for conservation of evaporated phase of liquid droplets and species of the gas phase are also solved on the 
Eulerian mesh. The liquid droplet dynamics equations are solved on the Lagrangian frame. Details of the liquid droplet model will be presented in Section 2.2. The conservation equations in compact vector notation are given by

$$
\frac{\partial \mathrm{U}}{\partial t}+\nabla \cdot \mathbf{F}=\mathrm{S}
$$

where the vector $\mathrm{U}$ represents the primitive flow variables and $\mathbf{F}$ holds the convective and diffusive terms, and they are given below [23].

$$
\mathrm{U}=\left(\begin{array}{c}
\rho \\
\rho u_{1} \\
\rho u_{2} \\
\rho u_{3} \\
\rho e \\
\rho Y_{k}
\end{array}\right), \quad F_{i}=\left(\begin{array}{c}
\rho u_{i} \\
\rho u_{i} u_{1}+P \delta_{i 1}-\mu A_{i 1} \\
\rho u_{i} u_{2}+P \delta_{i 2}-\mu A_{i 2} \\
\rho u_{i} u_{3}+P \delta_{i 3}-\mu A_{i 3} \\
\rho(\rho e+P) u_{i}-\mu A_{i j} u_{j}+q_{i} \\
\rho u_{i} Y_{k}-\rho \hat{u}_{i}^{k} Y_{k}
\end{array}\right) .
$$

Here, the density of the gas is represented by $\rho$ and the viscosity is given by $\mu$. $\mathbf{u}, e$ and $P$ represent the velocity, the total specific energy and the pressure, respectively. Species mass fraction and diffusion velocities of the $k^{\text {th }}$ species are given by $Y_{k}$ and $\hat{u}_{i}^{k}$, respectively. $\left(u_{1}, u_{2}, u_{3}\right)$ are the components of the velocity field $\mathbf{u}$ along the principal directions $1,2,3$. Definition of the total specific energy is given by the following equation

$$
e=\frac{P}{\gamma-1}+\frac{1}{2} u_{i} u_{i}
$$

where $\gamma$ is the ratio of the gas specific heat capacities. The heat flux $\mathrm{q}$ is given by

$$
\mathbf{q}=-\lambda \nabla T,
$$

in which $T \& \lambda$ represent temperature and thermal diffusivity, respectively. The diffusion velocity $\hat{\mathbf{u}}^{k} Y_{k}$ of the $k^{\text {th }}$ species is defined in terms of the species diffusivity $D_{k}$, the relationship is given by

$$
\hat{\mathbf{u}}^{k} Y_{k}=D_{k} \nabla Y_{k} \text {. }
$$

The source term in the governing equation arises due to the coupling with the liquid droplet model. The evaporation, heat exchange and momentum of the liquid droplets give rise to source terms to the mass, momentum, energy and droplet vapour species. The source term vector is given by

$$
S=\left(\begin{array}{c}
S_{\rho} \\
S_{\rho u_{1}} \\
S_{\rho u_{2}} \\
S_{\rho u_{3}} \\
S_{\rho e} \\
S_{\rho Y_{k}}
\end{array}\right)
$$

The terms $S_{\rho}, S_{\rho \mathbf{u}}$ and $S_{\rho e}$ are the source terms to the mass, momentum and energy equations. Of the species source terms $S_{\rho Y_{k}}$, the non-droplet vapor species are zero. These terms will be discussed in detail in the following section.

\subsection{Droplet Model}

The single droplet model has been widely used in the literature for modeling the evaporation and dispersion of liquid droplets. In this approach, the evaporation and transport of each droplet are tracked separately. While the droplet-droplet interactions are most often ignored, the model focuses primarily on the interaction of each droplet with the bulk gas phase and solid surface/geometries[13]. The equations governing the transport, dispersion and settling of the droplets are presented below.

$$
\begin{aligned}
\frac{d \mathbf{x}_{d}}{d t} & =\mathbf{u}_{d}, \\
\frac{d \mathbf{u}_{d}}{d t} & =\frac{3 C_{d}}{4 d_{d}} \frac{\rho}{\rho_{d}}\left(\mathbf{u}-\mathbf{u}_{d}\right)\left|\mathbf{u}-\mathbf{u}_{d}\right|, \\
C_{d} & = \begin{cases}\frac{24}{R e_{d}}\left(1+1 / 6 R e_{d}^{2 / 3}\right) & R e_{d}<1000, \\
0.424 & R e_{d}>1000,\end{cases} \\
R e_{d} & =\frac{\rho\left(\mathbf{u}-\mathbf{u}_{d}\right) d_{d}}{\mu} .
\end{aligned}
$$

In the above equations, $\mathbf{u}_{d}$ is the droplet velocity, $\mathbf{x}_{d}$ is the droplet position, the droplet drag coefficient $C_{d}$ is expressed a function of the droplet Reynolds number $R e_{d}$ and $d_{d}$ is the droplet diameter. While a general expression for droplet drag coefficient has been presented above, in the application of interest in this work, i.e. sputum droplet dispersion, the droplet Reynolds numbers for the majority of the droplets are expected to be within 1000 .

The evaporation of the droplet is modeled by the energy balance equation to track the droplet surface temperature and the evaporation mass flux equation to track the mass of the droplet, which are given below.

$$
\begin{aligned}
\frac{d T_{d}}{d t} & =\frac{N u}{3 P r} \frac{c_{p}}{c_{l}} \frac{f_{1}}{\tau_{d}}\left(T-T_{d}\right)+\frac{1}{m_{d}}\left(\frac{d m_{d}}{d t}\right) \frac{L_{V}}{c_{p, d}}, \\
\frac{d m_{d}}{d t} & =\dot{m}_{d} .
\end{aligned}
$$

Here, $m_{d}$, and $T_{d}$ are the mass and temperature, respectively. $T$ is the temperature of the ambient air, $L_{v}$ the latent heat of evaporation at the droplet temperature. $c_{p}$ and $c_{l}$ are the specific heat at a constant pressure of the ambient air and the specific heat capacity of the liquid droplet, and $\tau_{d}$ is the response time of the droplet. $f_{1}$ is a correction to the heat transfer due to evaporation of the droplet[1, 21]. Nu \& Pr are the Nusselt and Prandtl numbers, respectively. For the evaluation of the Prandtl number the standard definition is used, whereas the Nusselt number [24,25] is evaluated using the expression is given below along with the definition of $\tau_{d}$.

$$
\begin{aligned}
\tau_{d} & =\frac{\rho_{d} d_{d}^{2}}{18 \mu}, \\
N u & =2+0.552 \operatorname{Re}_{s}^{1 / 2} \operatorname{Pr}^{1 / 3} .
\end{aligned}
$$

The slip Reynolds number which is defined in terms of the slip velocity of a droplet with respect to the gas phase flow velocity, 
$\mathrm{u}_{\mathrm{slip}}=\max \left(\left|\mathbf{u}-\mathbf{u}_{\mathrm{d}}\right|\right)$, is given by

$$
R e_{s}=\frac{\rho \mathrm{u}_{\mathrm{slip}} \mathrm{d}_{\mathrm{d}}}{\mu} .
$$

In order to evaluate the evaporation mass flux, the non-equilibrium Langmuir-Knudsen model[18] employed in this work. The time rate of change of the mass of the droplet is given by the following equation.

$$
\dot{m}_{d}=-\frac{m_{d}}{\tau_{d}}\left(\frac{S h}{3 S c}\right) \ln \left(1+B_{M}\right),
$$

in which $S h, S c \& B_{m}$ are the Sherwood number, Schmidt number and the mass transfer number, respectively. The expression for Sherwood number and the mass transfer are given below.

$$
\begin{aligned}
& s h=2+0.552 R e_{s}^{1 / 2} S c^{1 / 3}, \\
& B_{m}=\frac{Y_{V, s}-Y_{V}}{1-Y_{V, s}} .
\end{aligned}
$$

As can be noted from the above equation, the mass transfer number is function of the liquid droplet's vapor mass fraction at the far field, $Y_{V}$ and that at the droplet surface, $Y_{V, s}$. The droplet surface vapor mass fraction in turn is given by

$$
Y_{V, s}=\frac{X_{V, s}}{X_{V, s}+\left(1-X_{V, s}\right) \bar{W} / W_{V}} .
$$

Here, $\bar{W} \& W_{V}$ are the average molecular weight of all the species in ambient fluid and the molecular weight of the liquid fuel vapour, respectively.

The mole fraction of the droplet vapor at the droplet surface is given by

$$
\text { with } \quad X_{V, s}=\frac{P_{s a t}}{P}-\left(\frac{2 L_{k}}{d_{d}}\right) \beta \text {. }
$$

In turn, the droplet vapor mass fraction is a function of the saturated vapor pressure $P_{s a t}$, the pressure of the ambient fluid, nondimensional evaporation parameter $\beta$ based on the Langmuir-Knudsen evaporation law and $L_{k}$ is the Knudsen layer thickness which are given by

$$
\begin{aligned}
& \beta=-\left(\frac{\rho_{d} P r}{8 \mu}\right) \frac{d d_{d}^{2}}{d t}, \\
& L_{k}=\frac{\mu\left\{2 \pi T_{d}\left(R / W_{F}\right)\right\}^{1 / 2}}{S c P},
\end{aligned}
$$

where, $R$ is the universal gas constant.

Based on the heat and mass exchange of the droplets with the ambient fluid, and the momentum of the droplet we can summarize the arising source terms to the gas phase governing equations as follows.

$$
\begin{aligned}
& S_{\rho}=-\frac{1}{\Delta V} \sum_{n} \frac{d m_{d}}{d t}, \\
& S_{\rho u}=-\frac{1}{\Delta V} \sum_{n} \frac{d m_{d} u_{d}}{d t}, \\
& S_{\rho e}=-\frac{1}{\Delta V} \sum_{n} \frac{d m_{d} h_{d}}{d t}, \\
& S_{\rho Y_{k}}=-\frac{1}{\Delta V} \sum_{n} \frac{d m_{d, k}}{d t} .
\end{aligned}
$$

As the droplets sizes can be significantly smaller than the spacing on Eulerian mesh, we adopt a Particle-Source-In-Cell (PSI-Cell) approach to sum the contribution of all the droplets that may be present in one fluid control volume. In the preceding set of equations, $n$ is the number of droplets in one control volume of the Eulerian mesh, $\Delta V$ is the volume of the control volume, $m_{d}$ is the mass of a given droplet, $m_{d, k}$ is the fraction of $m_{d}$ corresponding to species $k, \mathbf{u}_{d}$ is the droplet velocity, and the specific enthalpy of the fuel droplet is given by $h_{d}$.

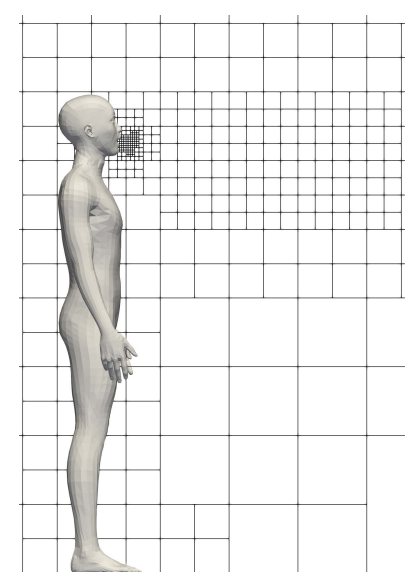

(a)

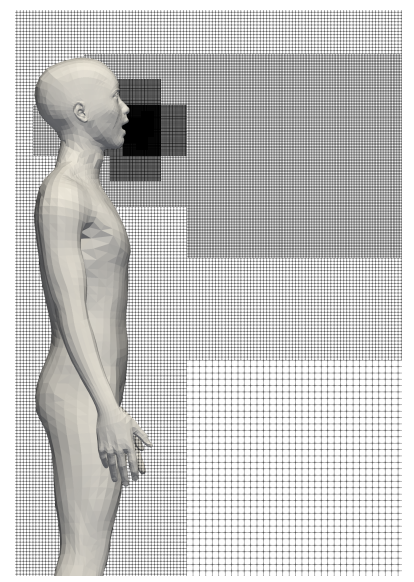

(b)
Figure 1: a) Discretization of a computational domain into cubic blocks. b) Subdivision of cubes into cells.

\subsection{Building Cube Method}

Modelling large scale dispersion of droplets requires modelling fluid flow over large spatial regions involving complex terrain and geometries. This poses two challenges from a computational standpoint. First, employing conventional body-fitted grids makes mesh generation around complex geometries a tedious and time-consuming task. On the other hand, usage of structured Cartesian meshes can blow up the mesh size by $\mathrm{O}\left(N^{3}\right)$ as the domain size or resolution is increased. The second challenge is the efficiency of parallelization, domain decomposition of Body Conformal Grids (BCG) is not straightforward and may not always yield the best results. These limitations can be overcome using hierarchical block-structured 
Cartesian meshing approaches $[5,10,20]$. In this work, we adopt a meshing methodology known as Building Cube Method (BCM) [20] to model the Eulerian mesh in this work.

In $\mathrm{BCM}$, the computational domain is first decomposed into discrete blocks know as cubes. While in principle these blocks can have edges of different sizes, in this work, and most applications of $\mathrm{BCM}$, the blocks are designed to have equal sides. Consequently, the moniker cube is used for the blocks. An example of a cubic decomposition is shown in Fig. 1a. As can be seen in the figure, depending on the problem requirement, where necessary, larger cubes are successively subdivided into finer cubes giving rise to a hierarchy of smaller cubes over layers of larger cubes. Refinement of larger cubes can be carried out such the ratio of the size of successive large and small cubes can be arbitrary. However, in this work, this refinement ratio is restricted to a constant value of two as it reduces the complexity of programming. And, refinement ratios larger than two can add substantial numerical dissipation when propagating information from fine to coarse meshes. Thereafter, every cube is further discretized to generate cells or fluid control volumes (Fig. 1b), on which the discrete governing equations are solved, such that irrespective of the size of a cube every cube has the same number of cells. This implies that whether a given cube is on the coarsest level of decomposition or the finest, the computational load of every cube is the same. Consequently, the cubic unit of the computational domain is treated as the fundamental computational unit for domain decomposition during parallel computing leading to excellent scalability. Time dependent dynamic mesh refinement and coarsening is usually adopted in hierarchically structured meshes to track unsteady flow phenomenon, moving geometries, dispersing droplets, etc. However, the current framework lacks this capability. This limitation can be alleviated, at the cost of larger meshes, by carefully designing the mesh keeping in mind the specific target and the expected flow physics.

\subsection{Lagrangian model}

To model the dispersion of sputum droplets in real-world conditions it is necessary to include and model the effects of solid geometries on the fluid flow and consequently on the droplet dispersion. To that end, we adopt a discrete forcing immersed boundary method to model solid geometries immersed in the fluid[2, 4, 15, 19]. IB methods are attractive due to their ability to robustly model highly complex geometry with minimal or no effect on the mesh generation process. At the implementation level, to impose the IB boundary conditions at every fluid cell with which an IB intersects, the IB is discretized into a discrete set of Lagrangian marker particles down to the level of the spacing of the fluid cell with which it intersects.

As has already been briefly discussed in Section 2.1, the Lagrangian marker particles approach is also used to model the sputum droplets. In this approach, a collection of the same sized droplet is grouped in an entity known as a computational parcel. Thereafter, a computational parcel is modelled as one Lagrangian marker particle. There are different strategies used in the literature to define the computational parcel. In one of the approaches, a single diameter is assigned to all computational parcel (modelled as a sphere), the parcel is then populated with liquid droplets of a given size until the total sum of the volume of the droplets added to the parcel is equal
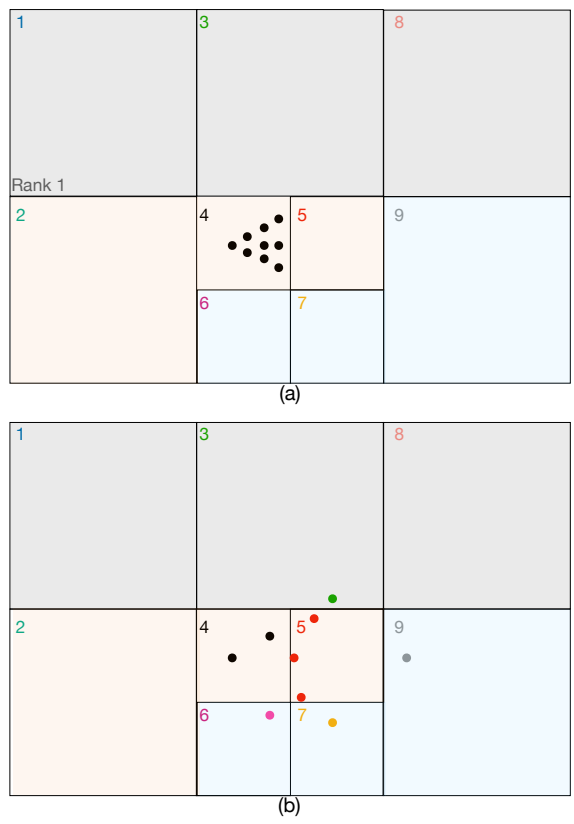

Figure 2: Cubes of a sample BCM mesh decomposed across three MPI process ranks 1 to 3 (color coded accordingly). Allocation of Lagrangian marker particles to respective sets as they move from cube with index 4 (top) to cubes with indices $3,5,6,7,9$.

to the volume of the computational parcel. Therefore, the number of droplets in each parcel can vary depending on the diameter of the droplet. In an alternate approach, the number of droplets in each parcel is fixed. In this work, both strategies are employed depending on the application.

As the IB geometries are discretized to the same extent as the mesh in which they reside, the total count of the Lagrangian marker particles for surfaces of IB can be $\mathrm{O}\left(N^{2}\right)$. And, depending on the application and modelling requirements, the total number of droplets and consequently the number of Lagrangian marker particles, can be $\mathrm{O}\left(10^{7}\right)$ [30]. More importantly, as the indexing of BCM mesh's cube blocks is arbitrary identifying the cube in which a given Lagrangian marker particle lies would involve search operations, which should be avoided when possible.

Given these factors, care must be taken in developing the Lagrangian data structure to optimize computational performance. For this, we employ the same strategy of discretization as is used in the BCM mesh. During initialization, the Lagrangian marker particles are grouped into sets wherein the sets are defined to match the underlying cube data-structure of the BCM mesh. Each set of the Lagrangian data structure is defined such that only those Lagrangian marker particles that are within the corresponding cube of the BCM are assigned to the set. This is illustrated in Fig. 2. Each cube and the corresponding set of a sample mesh are indexed from 1 to 9 in the figure. In Fig. 2a the Lagrangian marker particles are located within the cube indexed 4, so they are assigned to set(4), highlighted by the colour corresponding to this set. As the Lagrangian particles cross cube boundaries, when they move, 
they are removed from the previous set and assigned to the set corresponding to the cube into which they have entered. This illustrated in Fig. $2 \mathrm{~b}$ by colouring the particles with the colour of the set to which they belong. This insertion and removal of Lagrangian particles is efficiently carried out using the particle's global index based hash table. With regards to the domain decomposition for parallel computing, a spatial decomposition strategy is employed in this work[14]. The computational domain is decomposed such that on any given partition the Eulerian BCM mesh and the Lagrangian marker particles spatially overlap. This approach often leads to load imbalance across partitions which needs to be addressed with suitable load balancing strategies. However, in this work, we have not adopted any load balancing strategies. Nonetheless, we find that the present framework yields good parallel performance (see Section 4.6). Details of parallel implementations of the present work can be found in the literature[11, 12, 22].

The current framework allows arbitrary motions of Lagrangian marker particles, arising from the IB discretization and the computational parcels of sputum droplets, throughout the BCM mesh across coarse and fine regions. As discussed in Sec. 2.3, we do not employ dynamic mesh refinement in the present implementation of BCM. Therefore, when Lagrangian marker particles of the IB move from finer to coarser mesh region a corresponding loss in accuracy is expected. This limitation can be addressed by designing the $\mathrm{BCM}$ with the required level of mesh resolution in the expected path of IB geometries. As we employ a Particle-In-Cell (PIC) approach, the movement of the droplet's computational parcel from fine to coarse or vice-verse does not affect the evaluation of source term due to the droplet model. On the other hand, the droplet's motion and evaporation dynamics would be affected by the loss of accuracy of the flow field in the coarse mesh region.

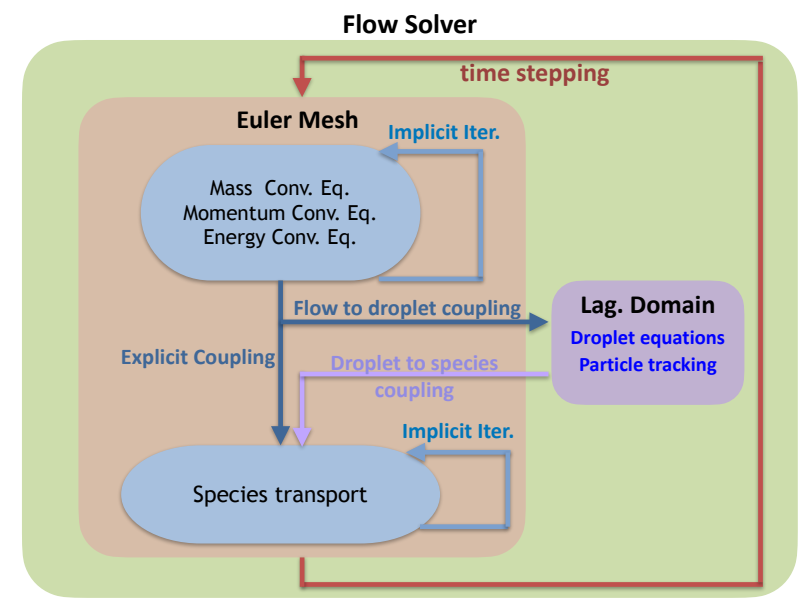

Figure 3: A schematic of the flow solver for the sputum droplet modeling.

\subsection{Droplet-fluid coupling for sputum droplet model}

To study the role of droplet dispersion in spreading diseases over large spatial distances it is necessary to simulate the dispersion of

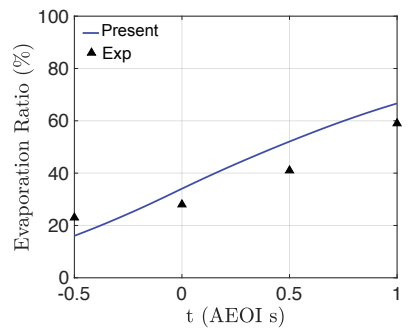

(a)

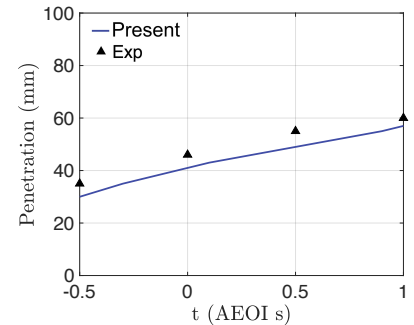

(b)
Figure 4: (a) Ratio of mass of vapour phase of the droplets to that liquid phase of the droplets. (b) A comparison of the penetration of the vapour phase of the spray After the End Of Injection (AEOI) with experimental measurement.

droplets over a long duration of time. Fully explicit time integration algorithms for the compressible Navier-Stokes equations require a time step of the order of $10^{-6} \mathrm{~s}$ or lower depending on the mesh spacing. This makes long-duration simulations impractical. On the other hand, implicit algorithms allow for arbitrarily large time steps making long-duration simulations feasible. However, implicit algorithms for coupled fluid flow and species transport equations are fairly complex, implementation of which can be cumbersome. As an alternative, we adopt a split time integration strategy in which the temporal integration of fluid flow equations and the species transport equations are split. Separate implicit temporal algorithms are adopted for the fluid flow and species transport equations. This approach is feasible if there is no strong coupling between the fluid flow and species transport equations. Therefore, for the sputum droplet modelling, we alter the coupling mechanism and adopt a weak one-way coupling between the two. In this approach the species transport is affected by the flow, however, the species concentration does not influence the fluid flow. Furthermore, to ensure numerical stability of the effect of droplet evaporation is added only to the species transport equations while the droplet source term to the fluid flow equations is ignored. The modified source term is given by $\mathrm{S}=\left[0,0,0,0,0, \mathrm{~S}_{\rho \mathrm{Y}_{\mathrm{k}}}\right]$. A schematic of flow solver with the one-way coupling for sputum droplet modelling is shown in Fig. 3. This type of one-way coupling is commonly used for modeling sputum droplet dispersion because the contribution by droplets to fluid flow in the form of momentum and energy are expected to be negligible[16, 26, 29].

\section{SIMULATION ENVIRONMENT}

All the simulations presented in this work, except for the validation case, were carried out on the Fugaku supercomputer. The validation simulation was carried on an Intel CPU based desktop PC. Fugaku comprises 158,976 nodes. Each node is equipped with a Fujitsu A64FX processor, which consists of 48 compute cores and 4 additional cores, and a memory of $32 \mathrm{GiB}$. The nodes are interconnected with 28Gbps, 2 lanes, 10 port TofuD interconnect. 


\section{RESULTS}

\subsection{Validation}

To validate the numerical framework presented in this work we simulated P-Xylene liquid droplet spray into a quiescent ambient flow and compare it with experimental data available in the literature[27]. For this validation, the full two-way coupling model with an explicit solver presented in Section 2.1 is used. The conditions under which the experiments were carried out are as follows: a quantity of $1.68 \mathrm{mg}$ liquid P-xylene was injected through a nozzle of diameter $15 \mu \mathrm{m}$ for a duration of $1.51 \mu \mathrm{s}$ in ambient fluid consisting of Nitrogen at $500 \mathrm{~K}$ at $1 \mathrm{MPa}$. A computational domain extending from -50 to $50 \mathrm{~mm}$ along with the lateral directions, 0 to $-400 \mathrm{~mm}$ was used for this simulation. The liquid droplets are injected vertically downwards at the origin. The $\chi^{2}$ distribution was used for the droplet diameter and the mean computational parcel diameter was set to $25 \mu \mathrm{m}$. The mesh resolution in the path of the droplet trajectory was $1.5 \mathrm{~mm}$ up to a distance of $100 \mathrm{~mm}$ from the point of droplet injection.

In this validation, the bulk evaporation and transport of the droplets of the simulation are compared with experimental data. The is an ideal candidate for validating the two key components of the droplet model namely the evaporation model and the transport model. By comparing the evaporation ratio of the simulation result with experimental data, the evaporation model is validated. Evaporation ratio is the ratio of the total mass of the vapour phase of the droplet to the total mass of the liquid phase of the droplets in the domain. The comparison of evaporation ratio as a function of time After the End Of Injection (AEOI) in Fig. 4(a) shows reasonable qualitative and quantitative agreement with experimental data. The drag model of the droplet modelling is validated in Fig. 4(b) by comparing the distance traversed by the vapour phase of the droplet with experimental data.

\subsection{Sputum droplet modeling parameters}

Before proceeding to the main results of simulations on droplet dispersion, the modeling parameters, boundary and initial conditions necessary for such simulations will be presented in this section. To model sputum droplet dispersion three primary input parameters are necessary - a) distribution of droplet diameter at the exit of the mouth and the total quantity of saliva or a count of droplet number that is being ejected, b) flow conditions at the mouth exit during a speech, a cough or a sneeze, c) dimensions of the mouth opening in the particular expiration event. While flow and droplet dynamics during human cough are extensively studied in the literature $[9,30,31]$, all the aforementioned parameter requirements are not met by one single study. Moreover, the studies on flow and droplet statistics during speech are limited $[6,8]$. Therefore, in this work data from various studies are sourced to model the droplet dispersion of cough. The droplet diameter distribution adopted in this work is shown in Fig. 5(a) [6, 7]. The flow rate and area of mouth opening of male subjects during a cough are taken from the work of Gupta and coworkers [9]. The flow velocity of a cough based on the flow rate and area is presented in Fig. 5(b). Another aspect of cough droplet modelling that is not available in the literature is the timing of the droplet ejection during cough. It is not clear whether all the droplets are ejected instantaneously, or over the

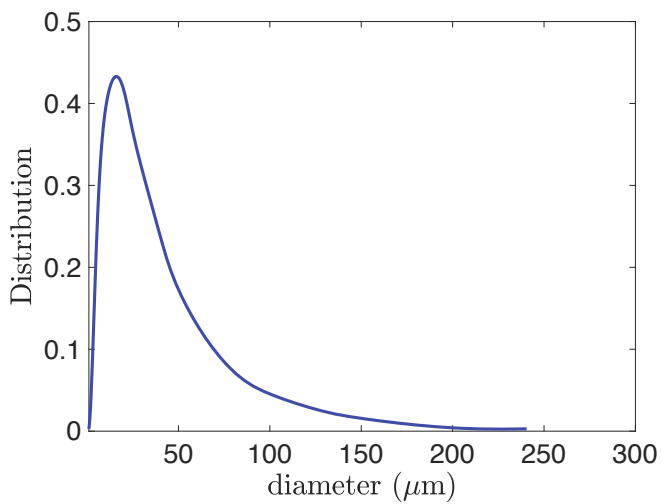

(a)

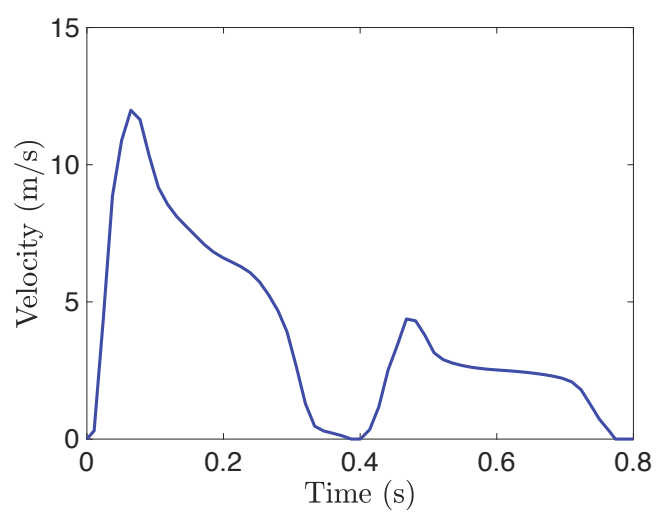

(b)

Figure 5: a) Distribution of droplet diameter at the time of injection[28]. b) The velocity profile of flow generated during the cough as a function of time[9].

span of duration for which a cough lasts. Therefore, due to lack of information, we assume that the instantaneous mass rate of the sputum ejection follows the same profile as the flow (Fig. 5(b)).

In the case of droplet dispersion during speech, information of flow and quantity of droplets ejected is limited in the literature. A reason for limited information on speech in the literature could be because determining the dynamics of flow and droplet ejection during speech is a highly subjective matter as it strongly depends on the language and cultural nuances of the individual who is speaking. Due to the lack of flow data in the literature, we adopt a squared sinusoidal flow rate, $\dot{v}=A_{i} \sin ^{2}\left(\pi t / T_{i}\right)$, to model the flow generated when counting from 1 to 10 in the English language. The peaks of the flow shown in Fig. 6(b) correspond to the amplitude $A_{i}$ and a variable period $T_{i}$ used for each word can be deduced from the plot. Experimental data on droplet diameter distribution during speech has been reported by several studies in the literature $[6,7,30]$. The droplet diameter distribution employed for modeling speech, shown in Fig. 6(a), is based on the distribution reported by [7] with minor alterations. As the overall droplet count for speech 
per word is small (80), for each word we have chosen to eject all the droplets instantaneously. The injections are uniformly spaced across the duration of speech of the 10 words $(5.65 \mathrm{~s})$. The final parameter necessary to close the problem is the area of the mouth during speech. An approximate measurement of one of the author's mouth opening when speaking was used to extract the mouth area, which was approximately measured to be $6 \mathrm{~cm}^{2}$.

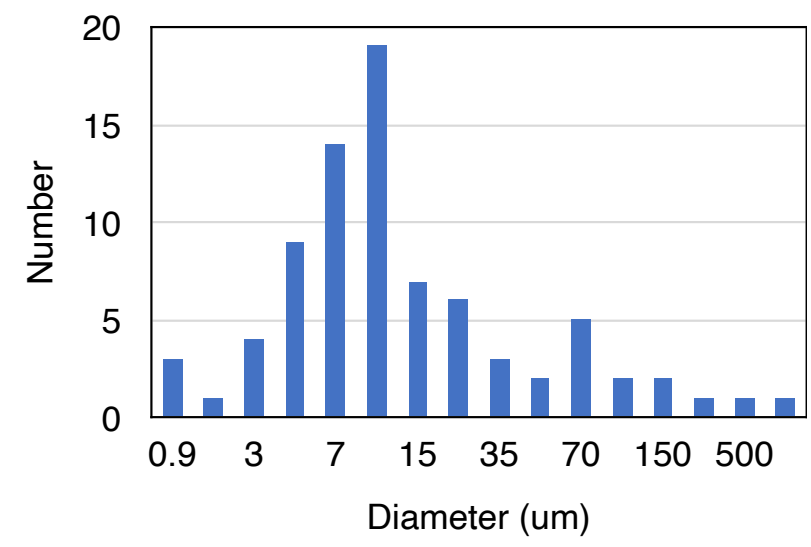

(a)

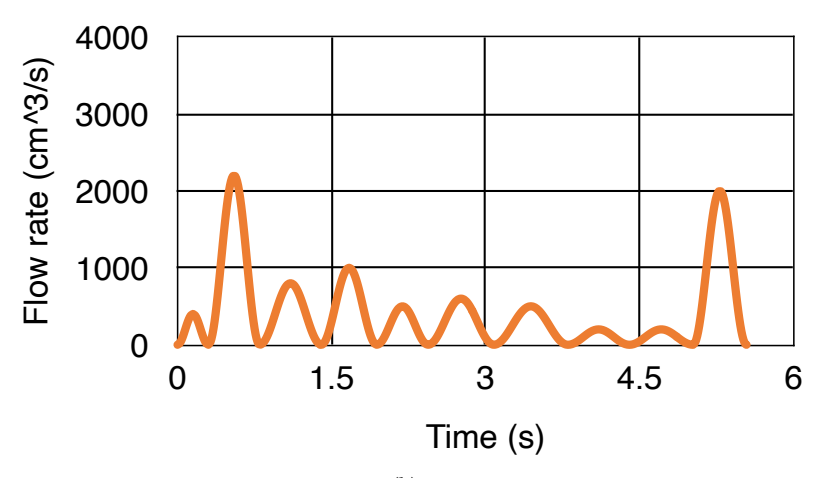

(b)

Figure 6: a) Diameter distribution of droplets ejected during speech. b) Profile of the flow generated from the mouth during speech.

\subsection{Boundary and initial conditions:}

For all cases presented in this work the ambient condition of the air was approximately set to STP conditions: Humidity, Temperature and pressure were set to $60 \%$ relative humidity, $297 \mathrm{~K}$ and $1 \mathrm{~atm}$, respectively. Stagnant ambient air is used as an initial condition for the simulations. The initial conditions of the droplets at the time of injection during a coughing event is as follows. The initial velocity, density, temperature of the droplets were chosen to be $0 \mathrm{~m} / \mathrm{s}, 1000$ $\mathrm{kg} / \mathrm{m}^{3}$ and $308 \mathrm{~K}$.

A circular mouth of area $4 \mathrm{~cm}^{2}$ [9] is used to model the mouth opening for cough simulations. For speaking simulations the mouth

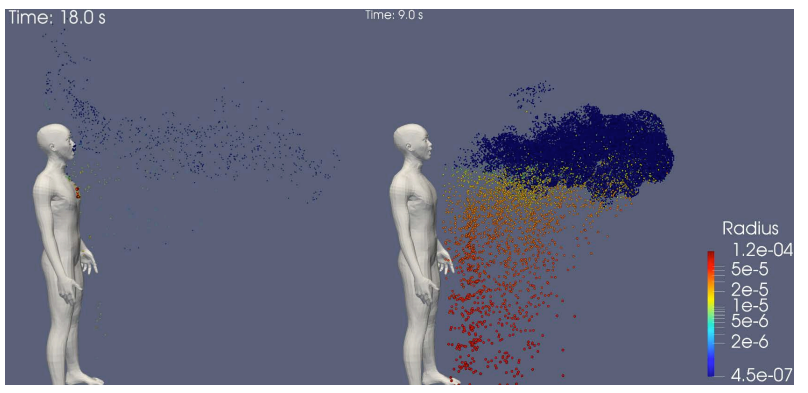

Figure 7: Left: Dispersion of sputum droplets after $18 \mathrm{~s}$ of speech. Right: Dispersion of droplet after a cough.

area was taken to be $6 \mathrm{~cm}^{2}$. The mouth model is placed approximately $1 \mathrm{~cm}$ in front of the mouth of the human models in all the simulations presented in this work. The cough or speech flow boundary condition is imposed through the mouth model. The droplets are randomly injected 3 grid cells in front of the mouth model with zero initial velocity. Relative humidity of $90 \%$ and temperature of $307 \mathrm{~K}$ was specified for the flow emanating from the mouth model to account for the higher humidity and temperature of the air exhaled through mouth and nose.

\subsection{Cough and speech in a quiescent environment}

The first case we consider is the investigation of the dispersion of droplets during cough and speech in an environment in which the ambient fluid is motionless. The setup consists of a person in a standing pose subject modelled to cough and speak. The height of the human model is $1.7 \mathrm{~m}$. The human model is placed in a computational domain measuring $16 \times 16 \times 16 \mathrm{~m}^{3}$ with the feet of the human model coinciding with the centroid of the computational domain. A no-slip and isothermal boundary condition with $T=307 \mathrm{~K}$ is imposed on the human model through IB modelling. The slip boundary condition is applied to the computational domain boundaries. The numerical mesh used for this simulation is presented in Fig. 1. In the region where the cough flow emanates and where the droplets are injected into the domain, a mesh resolution is $0.5 \mathrm{~mm}$, which is rapidly decreased to $4 \mathrm{~mm}$. The $4 \mathrm{~mm}$ mesh spacing is extended over a distance of $1 \mathrm{~m}$ in front of the mouth in the expected path of the sputum droplets. The mesh is made up of 4593 cubes and $18,812,928$ cells.

A visualization of the droplet dispersion after a cough and during speech is shown in Fig. 7. It is seen that large droplets start settling under the influence of gravity and don't travel very far in both cases. However, medium and small-sized droplets manage to travel longer distances. Particularly, the aerosolized droplets are not influenced by the flow and travel as far as the flow carries them. In Fig. 8, the maximum distance traversed by the droplet in the front and lateral directions is presented. For the speaking case, the droplets traverse about $1.74 \mathrm{~m}$ and $0.71 \mathrm{~m}$ along with the front and lateral directions. In the cough case, these values are $1.98 \mathrm{~m}$ and $1.09 \mathrm{~m}$ in the front and lateral directions. 


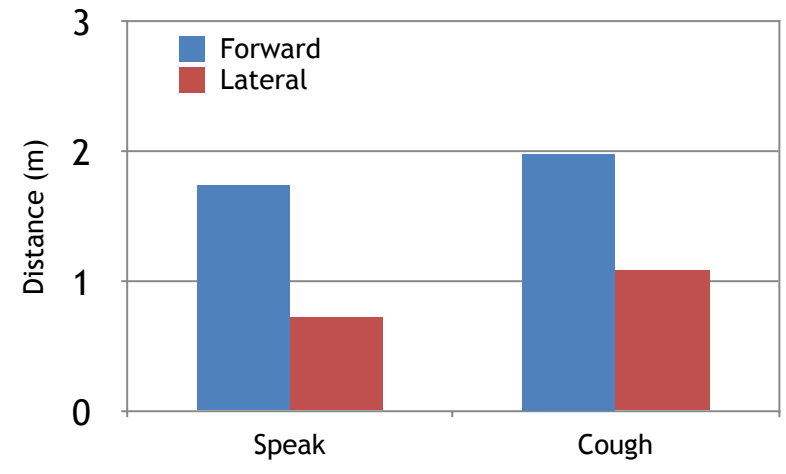

Figure 8: Distance traversed by the droplets 18s after the initiation of cough and speak expiration events in forward and lateral direction.

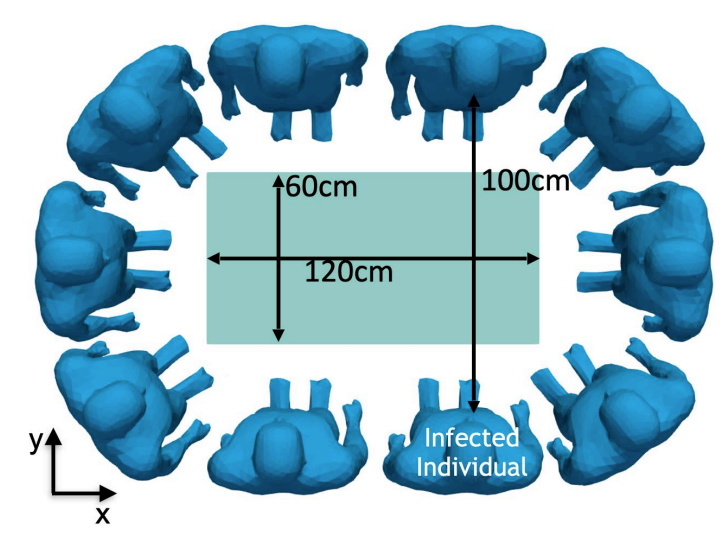

Figure 9: Geometry of the setup adopted for analysis of outdoor droplet dispersion

\subsection{Droplet dispersion in an outdoor environment}

We consider the dispersion of droplets in a real-world outdoor environment for the second application. The setup consists of a group of 10 people huddled around a barbecue table. One out of the 10 individuals assumed to be infected with a disease, such as COVID19 , that can be transmitted through respiratory droplets. The setup is shown in Fig.9. The dimensions of the table between the human models and the distance between the models are shown in the figure. All the human models are identical and measure $1.7 \mathrm{~m}$ in height. A relatively large computational domain is employed to model the effects of an outdoor environment. The computational domain extents are $-250 \mathrm{~m}$ to $250 \mathrm{~m}$ along the $\mathrm{x}$, and $\mathrm{y}$ directions, and from $-50 \mathrm{~m}$ to $200 \mathrm{~m}$ along the $\mathrm{z}$-direction. The vertical direction is along the $\mathrm{z}$-axis with gravity acting along the negative $\mathrm{z}$-axis. The centroid of the computational domain coincides with the feet of the infected person. A rectangular planar geometry measuring $50 \times 50 \mathrm{~m}^{2}$ with its normal along the $\mathrm{z}$-axis is used to model the ground. The centre of the ground geometry is aligned with the centroid of the computational domain. At the location of the mouth of the infected

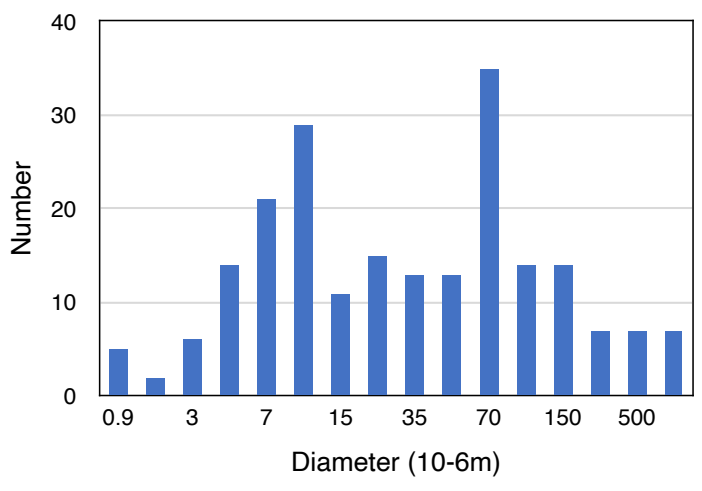

Figure 10: Droplet diameter distribution for speech at louder volume in outdoor environment.

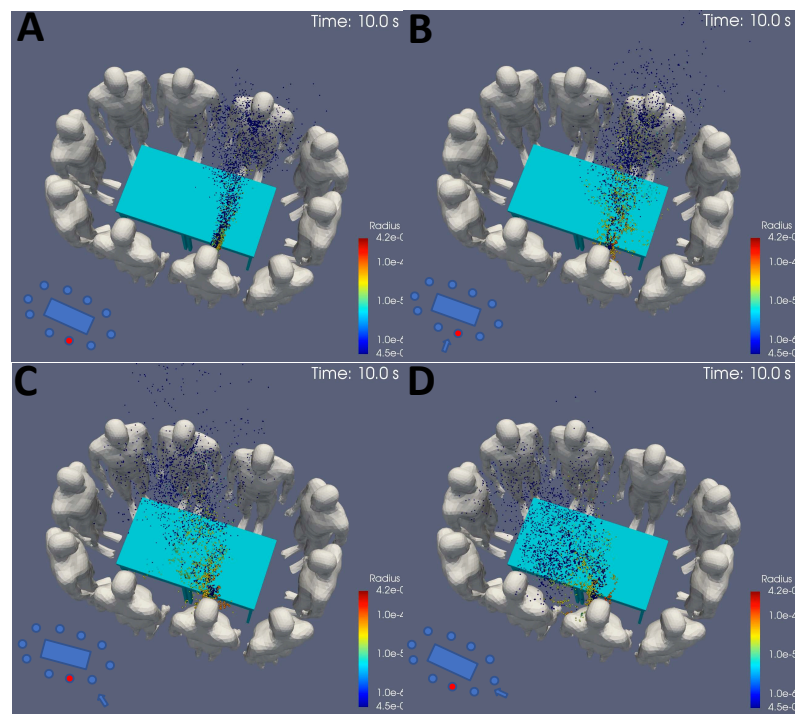

Figure 11: Visualization of droplet dispersion under A) no wind, and wind velocity $B) \mathbf{u}=(0,0.5,0) \mathbf{m} / \mathbf{s}$, C) $\mathbf{u}=$ $(-\sqrt{1 / 2}, \sqrt{1 / 2}, 0) \mathbf{m} / \mathbf{s}, \mathbf{D}) \mathbf{u}=(-0.5,0,0) \mathbf{m} / \mathbf{s}$

person, the mesh spacing is $1 \mathrm{~mm}$ which progressively increases to $16 \mathrm{~mm}$ in the spatial region around the table. The mesh spacing on all the human geometries and the table is $16 \mathrm{~mm}$. At all other regions, the mesh is progressively coarsened to contain the overall mesh size. The mesh was made up of 9552 cubes and 39,124,992 cells.

In an outdoor barbecue type environment, individuals would likely tend to speak with a louder voice. A consequence of this is that the overall flow generated at the mouth is likely to be higher. To account for this we increase the flow rate for the speaking case (see Fig. 6(b)) by 50 percent. Furthermore, a tendency commonly observed when speaking loudly is also an increase in the number and size of the sputum droplets being ejected. Therefore, we also alter the droplet diameter distribution model presented in Fig. 6(a). The new droplet diameter distribution model is shown in Fig. 10. 

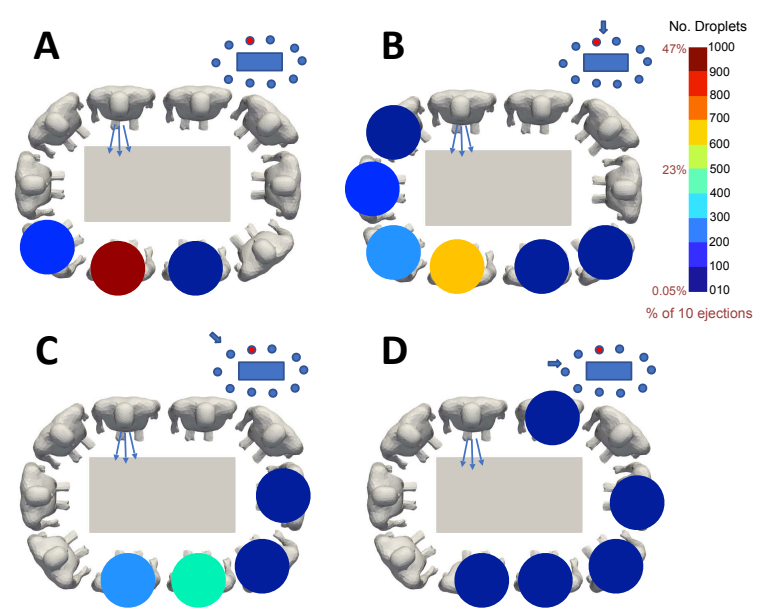

Figure 12: Quantitative evaluation of risk of each individual around the infected person.

The droplet dispersion was investigated under the conditions of no wind and varying wind direction: $\mathrm{A})$ no wind, $\mathrm{B}) \mathbf{u}=(0,0.5,0)$ $\mathrm{m} / \mathrm{s}, \mathrm{C}) \mathbf{u}=(-\sqrt{1 / 2}, \sqrt{1 / 2}, 0) \mathrm{m} / \mathrm{s}$, and D) $\mathbf{u}=(-0.5,0,0) \mathrm{m} / \mathrm{s}$. Before the initialization of the speaking model in the simulation, the simulation was carried out for a duration of $5 \mathrm{~s}$ to develop the flow due to natural convection around the human model. Thereafter, the speaking model was initialized during which the droplets are continuously injected according to the droplet model until the end of the simulation. A visualization of the droplet dispersion under different wind conditions is shown in Fig. 11. The direction of droplet dispersion is strongly dependent on the wind direction. And, depending on the direction of the wind, the individuals who might be exposed to the virus-laden droplets varies.

To assess the risk of infection of an individual upon exposure to the droplets, the total number of droplets that might be within the vicinity of an individual was measured. For this, the breathing zone around the head of each human subject was defined. The total number of droplets within the breathing zone of each individual was measured at an interval of $1 \mathrm{~s}$ after the initialization of the speaking model for a duration of 20 s (i.e. 20 measurements in total). The maximum value of these measurements for each subject was quantified as the risk. This quantity is presented in Fig. 12. In case A, only three subjects directly in front of the infected person are at risk of infection. In case $\mathrm{B}$, the presence of the wind tends to disperse the droplets laterally, increase the number of subjects being exposed to the droplets. In cases $\mathrm{C}$ and $\mathrm{D}$, due to the change in the direction of the wind, the subjects who are at risk of infection change accordingly.

\subsection{Parallel performance}

Finally, we present an evaluation of the computational performance of the numerical framework presented in this work. As the numerical model is composed of a combination of an Eulerian and Lagrangian mesh, achieving good parallel performance can be challenging. Therefore, we set up a problem that includes all the three key components of the numerical framework, namely the Eulerian

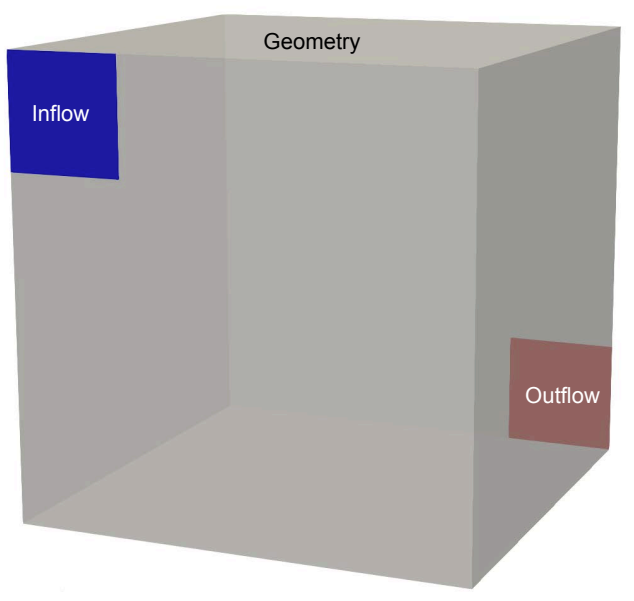

Figure 13: Setup of the geometry adopted for the scaling analysis.

Table 1: Mesh and CPU setup for weak scaling analysis.

\begin{tabular}{cccc}
\hline CPU & Cubes & Cells & Droplets \\
\hline 32 & 64 & 262144 & 4096 \\
256 & 512 & 2097152 & 32768 \\
2048 & 4096 & 16777216 & 262144 \\
16192 & 32768 & 134217728 & 2097152 \\
131072 & 262144 & 1073741824 & 16777216 \\
\hline
\end{tabular}

domain's flow solvers, Lagrangian domain's droplet solvers and the immersed boundary method for modeling complex geometries. The setup consists of $1.2 \times 1.2 \times 1.2 \mathrm{~m}^{3}$ computational domain in which a $1 \mathrm{~m}^{3}$ cubic box is placed to mimic a closed space. The box is modelled as an IB with no-slip and isothermal boundary condition. The box is fitted with inflow and outflow windows to model incoming and outgoing airflow through windows or air-conditioning systems (see Fig. 13). It should be noted that the wall of the box does not coincide with the computational domain boundary. The interior of the box is then uniformly populated with droplets. The total droplet count depends on the scaling analysis being carried out and will be mentioned later. The number of implicit iterations of the flow solver and the species solver is fixed across all the simulations to ensure uniform computation load for a given timestep. The simulations were carried out for 100 timesteps, and the total runtime was averaged for the 100 timesteps to obtain the runtime-per-timestep (RPT)

The mesh and Lagrangian particle setup for weak scaling is shown in Table 1 . To ensure that the computational load on all components of the numerical framework are increased proportionally, as the mesh size is increased by a factor of 8 for each data point of the weak scaling analysis, the number of droplets is proportionally increased. Furthermore, the Lagrangian particles that arise for the IB modelling of the box geometry automatically scale with the mesh 
size. The RPT for the weak scaling cases is presented in Fig. 14(a). The RPT increases initially when the number of CPUs is increased from 32 to 512, but, upon further increasing the CPU number, RPT is not significantly affected. This can also be noted from the plot of parallel efficiency for weak-scaling in Fig. 14(b). After the initial drop of efficiency to about $90.1 \%$ the efficiency slowly saturates to about $86.07 \%$ for the 131,072 CPU case.

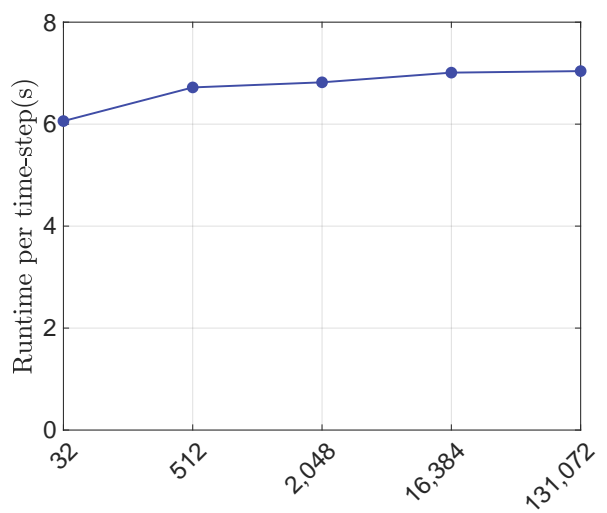

No. of procs

(a)

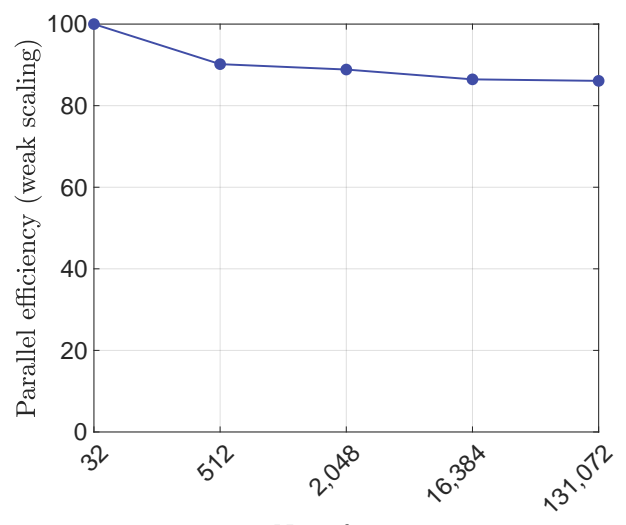

No. of procs

(b)

Figure 14: a)Runtime-per-timestep plotted again number of CPU. b) parallel efficiency of the weak scaling analysis plotted again number of CPU.

For the strong scaling analysis, we use the mesh and the setup that was used for the 131,072 CPU case in the weak scaling analysis. Keeping the mesh and the number of droplets $(16,777,216)$ constant, the number of CPU was increased from 8192 to 131,072 by a factor of 2 for each increment. The speed of the solver for one timestep compared to the 8192 CPUs case as the CPU number is increased is plotted in Fig. 15(a) and the parallel efficiency for the same is plotted in Fig. 15(b). We find that the overall speedup and parallel efficiency of the solver are good. In the highest CPU case, the parallel efficiency was $82.42 \%$

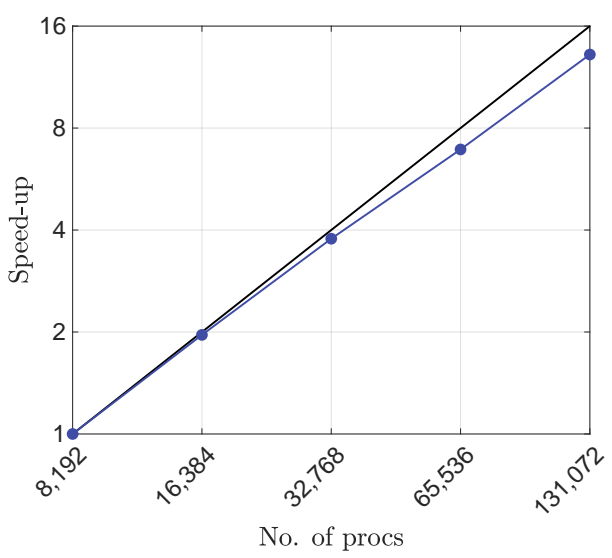

(a)

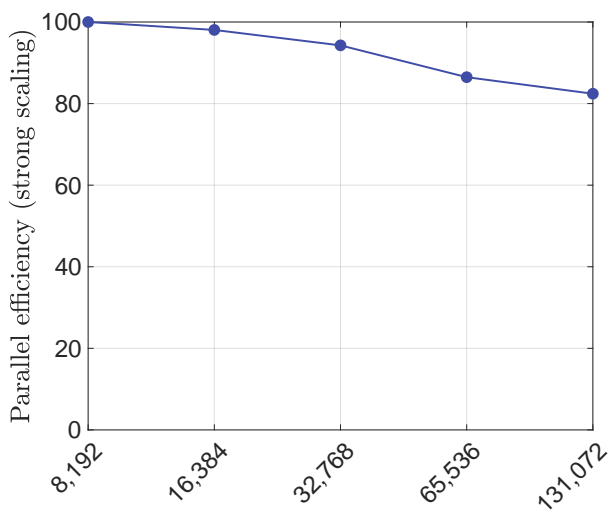

No. of procs

(b)

Figure 15: a)Speed up and b) parallel efficiency plotted against number CPU from the results of a strong scaling analysis.

\section{SUMMARY AND OUTLOOK}

In this work, we have presented a numerical framework developed for the simulation of large-scale dispersion of respiratory droplets in COVID-19 type pandemics. The numerical framework efficiently combined a fully compressible Navier-stokes solver and Lagrangian droplet dynamics model to enable large scale parallel simulation of droplet dispersion in a real-world environment. The numerical method was validated by carrying a two-way coupled simulation of evaporation and transport of $\mathrm{P}$-xylene liquid droplets. The capability of the numerical method was demonstrated through the simulations of droplet dispersion in a quiescent environment for droplets ejected during cough and speech of person in standing position. The method was also applied to droplet dispersion in the real-world environment through the simulation of dispersion of droplets during speak in a barbecue setup.

To ensure good parallel performance, the numerical framework was developed on a BCM meshing framework and a BCM based Lagrangian marker particle data structure was used. The scaling 
analysis showed very good performance of the solver in the weak as well as the strong scaling analysis, demonstrating that the numerical framework presented in this work is an excellent candidate for simulation of virus-laden droplet dispersion in very large public spaces like airports, train stations, shopping malls, concert halls, etc. This was partially demonstrated through the simulation of droplet dispersion in a large open outdoor environment around a group of people.

\section{ACKNOWLEDGMENTS}

This work was supported by JST CREST Grant Number JPMJCR20H7, Japan, and through the computing resources provided on the $\mathrm{Fu}-$ gaku supercomputer by the RIKEN Center for Computational Science and through the HPCI system research project (Project ID: hp200154).

\section{REFERENCES}

[1] Yuya Baba and Ryoichi Kurose. 2008. Analysis and flamelet modelling for spray combustion. Journal of Fluid Mechanics 612 (2008), 45-79.

[2] Rahul Bale, Neelesh A Patankar, Niclas Jansson, Keiji Onishi, and Makoto Tsubokura. 2020. Stencil Penalty approach based constraint immersed boundary method. Computers \& Fluids 200 (2020), 104457.

[3] Rahul Bale, Wei Hsiang Wang, Chung-Gang Li, Keiji Onishi, Kenji Uchida, Hidefumi Fujimoto, Ryoichi Kurose, and Makoto Tsubokura. 2020. A Scalable Framework for Numerical Simulation of Combustion in Internal Combustion Engines. In Proceedings of the Platform for Advanced Scientific Computing Conference. 1-10.

[4] Amneet Pal Singh Bhalla, Rahul Bale, Boyce E. Griffith, and Neelesh A. Patankar. 2013. A unified mathematical framework and an adaptive numerical method for fluid-structure interaction with rigid, deforming, and elastic bodies. F. Comput. Phys. 250 (2013), 446 - 476

[5] Carsten Burstedde, Lucas C. Wilcox, and Omar Ghattas. 2011. p4est: Scalable Algorithms for Parallel Adaptive Mesh Refinement on Forests of Octrees. SIAM fournal on Scientific Computing 33, 3 (2011), 1103-1133. https://doi.org/10.1137/ 100791634

[6] Christopher Yu Hang Chao, Man Pun Wan, Lidia Morawska, Graham R Johnson, ZD Ristovski, Megan Hargreaves, K Mengersen, S Corbett, Yuguo Li, Xiaojian Xie, et al. 2009. Characterization of expiration air jets and droplet size distributions immediately at the mouth opening. Journal of Aerosol Science 40, 2 (2009), 122 133.

[7] JP Duguid. 1946. The size and the duration of air-carriage of respiratory droplets and droplet-nuclei. Epidemiology \& Infection 44, 6 (1946), 471-479.

[8] Jan Gralton, Euan Tovey, Mary-Louise McLaws, and William D Rawlinson. 2011. The role of particle size in aerosolised pathogen transmission: a review. fournal of Infection 62, 1 (2011), 1-13.

[9] JK Gupta, C-H Lin, and Q Chen. 2009. Flow dynamics and characterization of a cough. Indoor air 19, 6 (2009), 517-525.

[10] Tobin Isaac, Carsten Burstedde, Lucas C. Wilcox, and Omar Ghattas. 2015. Recursive algorithms for distributed forests of octrees. SIAM fournal on Scientific Computing 37, 5 (2015), C497-C531. https://doi.org/10.1137/140970963

[11] Niclas Jansson, Rahul Bale, Keiji Onishi, and Makoto Tsubokura. [n. d.]. CUBE: A scalable framework for large-scale industrial simulations. The International fournal of High Performance Computing Applications ([n. d.]), 1094342018816377.

[12] Niclas Jansson, Rahul Bale, Keiji Onishi, and Makoto Tsubokura. 2017. Dynamic Load Balancing for Large-Scale Multiphysics Simulations. In High-Performance Scientific Computing, Edoardo Di Napoli, Marc-André Hermanns, Hristo Iliev, Andreas Lintermann, and Alexander Peyser (Eds.). Springer International Publishing, Cham, 13-23.

[13] Tomoaki Kitano, Jun Nishio, Ryoichi Kurose, and Satoru Komori. 2014. Effects of ambient pressure, gas temperature and combustion reaction on droplet evaporation. Combustion and Flame 161, 2 (2014), 551-564.

[14] ChihKuang Kuan, Jaeheon Sim, Ezeldin Hassan, and Wei Shyy. 2013. Parallel Eulerian-Lagrangian Method with Adaptive Mesh Refinement for Moving Boundary Computation. In 51st AIAA Aerospace Sciences Meeting including the New Horizons Forum and Aerospace Exposition. 370.

[15] Chung-Gang Li, Makoto Tsubokura, and Rahul Bale. 2016. Framework for simulation of natural convection in practical applications. International Communications in Heat and Mass Transfer 75 (2016), 52-58.

[16] Li Liu, Jianjian Wei, Yuguo Li, and A Ooi. 2017. Evaporation and dispersion of respiratory droplets from coughing. Indoor Air 27, 1 (2017), 179-190.

[17] Rainald Löhner, Harbir Antil, Sergio Idelsohn, and Eugenio Oñate. 2020. Detailed simulation of viral propagation in the built environment. Computational
Mechanics 66, 5 (2020), 1093-1107.

[18] RS Miller, K Harstad, and J Bellan. 1998. Evaluation of equilibrium and nonequilibrium evaporation models for many-droplet gas-liquid flow simulations. International fournal of Multiphase Flow 24, 6 (1998), 1025-1055.

[19] Rajat Mittal and Gianluca Iaccarino. 2005. Immersed boundary methods. Annu. Rev. Fluid Mech. 37 (2005), 239-261.

[20] Kazuhiro Nakahashi. 2003. Building-Cube Method for Flow Problems with Broadband Characteristic Length. In Computational Fluid Dynamics 2002. Springer Berlin Heidelberg, 77-81.

[21] Mariko Nakamura, Fumiteru Akamatsu, Ryoichi Kurose, and Masashi Katsuki. 2005. Combustion mechanism of liquid fuel spray in a gaseous flame. Physics of Fluids 17, 12 (2005), 123301.

[22] Koji Nishiguchi, Rahul Bale, Shigenobu Okazawa, and Makoto Tsubokura. 2019. Full Eulerian deformable solid-fluid interaction scheme based on building-cube method for large-scale parallel computing. Internat. 7. Numer. Methods Engrg. 117, 2 (2019), 221-248.

[23] Thierry Poinsot and Denis Veynante. 2005. Theoretical and numerical combustion. RT Edwards, Inc

[24] WE Ranz and W R Marshall. 1952. Evaporation from drops I. Chem. eng. prog 48, 3 (1952), 141-146.

[25] WE Ranz and W R Marshall. 1952. Evaporation from drops II. Chem. eng. prog 48, 3 (1952), 173-180

[26] John Redrow, Shaolin Mao, Ismail Celik, J Alejandro Posada, and Zhi-gang Feng. 2011. Modeling the evaporation and dispersion of airborne sputum droplets expelled from a human cough. Building and Environment 46, 10 (2011), 20422051.

[27] Kiyotaka Sato, Koji Shishime, Yoshitaka Wada, Kenta Kitamitsu, Masaharu Chato, and Keiya Nishida. 2012. Evaporation and Mixture Formation Characteristics of Ethanol and Gasoline Fuel Spray under High Temperature and High Pressure in D.I. Gasoline Engine. In Symposium (ILASS-fapan) on Atomization, Vol. 21. 46-53.

[28] Jianjian Wei and Yuguo Li. 2016. Airborne spread of infectious agents in the indoor environment. American journal of infection control 44, 9 (2016), S102-S108.

[29] X Xie, Y Li, ATY Chwang, PL Ho, and WH Seto. 2007. How far droplets can move in indoor environments-revisiting the Wells evaporation-falling curve. Indoor air 17, 3 (2007), 211-225.

[30] Gustavo Zayas, Ming C Chiang, Eric Wong, Fred MacDonald, Carlos F Lange, Ambikaipakan Senthilselvan, and Malcolm King. 2012. Cough aerosol in healthy participants: fundamental knowledge to optimize droplet-spread infectious respiratory disease management. BMC pulmonary medicine 12, 1 (2012), 1-12.

[31] Shengwei Zhu, Shinsuke Kato, and Jeong-Hoon Yang. 2006. Study on transport characteristics of saliva droplets produced by coughing in a calm indoor environment. Building and environment 41, 12 (2006), 1691-1702. 